\title{
Large-Eddy Simulation of Wind-Turbine Wakes: Evaluation of Turbine Parametrisations
}

\author{
Yu-Ting Wu • Fernando Porté-Agel
}

Received: 14 April 2010 / Accepted: 9 November 2010 / Published online: 14 December 2010

(C) Springer Science+Business Media B.V. 2010

\begin{abstract}
Large-eddy simulation (LES), coupled with a wind-turbine model, is used to investigate the characteristics of a wind-turbine wake in a neutral turbulent boundary-layer flow. The tuning-free Lagrangian scale-dependent dynamic subgrid-scale (SGS) model is used for the parametrisation of the SGS stresses. The turbine-induced forces (e.g., thrust, lift and drag) are parametrised using two models: (a) the 'standard' actuator-disk model (ADMNR), which calculates only the thrust force and distributes it uniformly over the rotor area; and (b) the actuator-disk model with rotation (ADM-R), which uses the blade-element theory to calculate the lift and drag forces (that produce both thrust and rotation), and distribute them over the rotor disk based on the local blade and flow characteristics. Simulation results are compared to high-resolution measurements collected with hot-wire anemometry in the wake of a miniature wind turbine at the St. Anthony Falls Laboratory atmospheric boundarylayer wind tunnel. In general, the characteristics of the wakes simulated with the proposed LES framework are in good agreement with the measurements in the far-wake region. The ADM-R yields improved predictions compared with the ADM-NR in the near-wake region, where including turbine-induced flow rotation and accounting for the non-uniformity of the turbine-induced forces appear to be important. Our results also show that the Lagrangian scale-dependent dynamic SGS model is able to account, without any tuning, for the effects of local shear and flow anisotropy on the distribution of the SGS model coefficient.
\end{abstract}

Keywords Actuator-disk model · Blade-element momentum theory · Large-eddy simulation · Wind-turbine wakes

Y.-T. Wu · F. Porté-Agel $(\varangle)$

School of Architecture, Civil and Environmental Engineering, École Polytechnique Fédérale de Lausanne, 1015 Lausanne, Switzerland

e-mail: fernando.porte-agel@epfl.ch 


\section{Introduction}

With the fast growing number of wind farms being installed worldwide, the interaction between wind-turbine wakes and atmospheric boundary-layer (ABL) turbulence, and its effects on energy production as well as dynamic loading on downwind turbines, have become important issues in both the wind energy and the atmospheric science communities (Vermeer et al. 2003). Understanding this interaction requires detailed knowledge of wind-turbine wakes and atmospheric turbulence at a wide range of spatial and temporal scales. Accurate prediction of the ABL flow and its interaction with wind turbines as well as turbine-induced wakes is of great importance in optimising the performance of wind turbines and wind farms. Particularly, it can be used to maximise the wind energy production and minimise the fatigue loading in the evaluation of wind farm layouts. In addition, numerical simulations can provide valuable quantitative insight into the potential impacts of wind farms on local meteorology.

During the last two decades, numerical modelling of wind-turbine wakes using computational fluid dynamics (CFD) has become increasingly popular (Sørensen and Kock 1995; Sørensen et al. 1998; Ammara et al. 2002; Sørensen and Shen 2002; Gómez-Elvira et al. 2005; Jimenez et al. 2007; Troldborg et al. 2007; Jimenez et al. 2008; Kasmi and Masson 2008; Ivanell et al. 2009). Despite the great potential of CFD to study wind-turbine wakes, there are still some open issues that need to be addressed in order to guarantee the accuracy of numerical simulations of high-Reynolds number boundary-layer turbulent flow in the $\mathrm{ABL}$ and in the turbine-induced wakes. For example, some of the existing numerical studies of wind-turbine wakes have investigated the structure and dynamics of the wakes of wind turbines placed in laminar free-stream flow (Sørensen and Kock 1995; Sørensen et al. 1998; Sørensen and Shen 2002; Ivanell et al. 2009). However, a recent wind-tunnel study (Medici and Alfredsson 2006) has shown that the structure of the wake of a model turbine located in the free-stream flow is substantially changed by the presence of turbulence in the flow. More recently, Chamorro and Porté-Agel (2009) carried out a wind-tunnel experiment to study the wake of a miniature wind turbine placed in a boundary layer developed over different surface roughness conditions. They showed that the incoming boundary-layer flow characteristics, which are affected by the surface roughness, have a strong effect on the turbine wake dynamics. Considering that wind turbines are always exposed to turbulent boundary-layer flow conditions, it is of interest to test the performance of numerical simulation techniques under such conditions.

Due to the high Reynolds number of the ABL flow, direct numerical simulation (DNS) is impossible, and numerical models require two important parametrizations: a turbulence model and a model for the turbine-induced forces. Previous studies of ABL flows through isolated wind turbines or wind farms have modelled the turbulence using a Reynolds-averaged Navier-Stokes (RANS) approach (Ammara et al. 2002; Alinot and Masson 2002; GómezElvira et al. 2005; Kasmi and Masson 2008) or a large-eddy simulation (LES) technique (Jimenez et al. 2007, 2008). In RANS, turbulent fluxes and energy are fully parametrized and, thus, the approach is incapable of predicting turbulent eddy motions. In contrast, LES explicitly resolves all scales of turbulent transport larger than the grid scale $\Delta$, while the smallest scales are parametrized using a subgrid-scale (SGS) model. Due to the fact that a large fraction of the turbulence is resolved, LES has become a popular tool for the study of turbulent transport in the ABL flows (e.g., Moeng 1984; Albertson and Parlange 1999; Porté-Agel et al. 2000; Chamecki et al. 2009).

A common parametrization strategy in LES consists of computing the SGS stresses with the Smagorinsky eddy-viscosity model (Smagorinsky 1963; Deadorff 1971; Porté-Agel et al. 2000), which requires the specification of the Smagorinsky coefficient. This is complicated 
by the dependence of the coefficient on local conditions such as distance to the ground, mean shear, and atmospheric stability. Evidence from both simulations and experimental a priori studies showed that the model coefficient should decrease in regions of the flow with large flow anisotropy at the smallest resolved and subgrid scales associated with large local mean shear and flow stratification (Deadorff 1980; Hunt et al. 1988; Horiuti 1993; Canuto and Cheng 1997; Porté-Agel et al. 2001b; Porté-Agel 2004). A major advancement in the LES-SGS modelling was the use of dynamic procedures (Germano et al. 1991; Lilly 1992), which is based on assuming the scale invariance of the model coefficient and using information from the smallest resolved scales to optimise the value of the model coefficient at every position and timestep without any ad hoc tuning. In order to guarantee numerical stability, these procedures require some kind of averaging. For cases of flow over complex topography, Meneveau et al. (1996) adopted the Lagrangian averaging in the dynamic model that computes the value of the model coefficient along fluid flow path without the need for spatial averaging. Porté-Agel et al. (2000) proposed the so-called scale-dependent dynamic model by relaxing the assumption of the scale invariance of the model coefficient on which the dynamic procedure relies. The simulation results showed that the dynamically computed coefficients are scale-dependent in simulations of the neutral boundary layer over homogeneous surfaces. The scale-dependent dynamic model was shown to overcome the limitations of the scale-invariant dynamic model in simulations of neutral ABL flow over flat surfaces.

Later, a Lagrangian scale-dependent dynamic model version was performed in simulations of neutral ABL flow over heterogeneous (Bou-Zeid et al. 2005; Stoll and Porté-Agel 2006) flat surfaces as well as flows over wave-like topography (Wan et al. 2007). Those studies showed that the Lagrangian scale-dependent dynamic model yields improved predictions of the flow statistics compared with the standard Smagorinsky and scale-invariant dynamic models.

In rotary aerodynamics, the generalized actuator disk and related models have commonly been used to parametrize turbine-induced forces in numerical investigations of wind-turbine wakes (Vermeer et al. 2003). These models define an actuator disk as a circular permeable rotor surface on which the turbine-induced forces (i.e., lift, drag and thrust) are parametrized and integrated over the spatial and temporal resolution. Note that this approach does not require resolving the boundary-layer flow around the turbine blade surface, which greatly reduces the computational cost requirements. The earliest approach used to parametrize the turbine-induced forces is based on the one-dimensional momentum theory. It leads to an overall thrust force, which acts in the axial direction and is uniformly distributed on the actuator disk. Since the resulting force acts only in the axial direction (i.e., no rotational component is considered), here we refer to this method as 'actuator-disk model without rotation' (ADM-NR). The ADM-NR has been used in numerical simulations of turbines in the context of a highly parametrized RANS technique (Gómez-Elvira et al. 2005). More recently, this approach has also been used in LES together with the traditional Smagorinsky model (Jimenez et al. 2007, 2008) or the Lagrangian scale-dependent dynamic model (Calaf et al. 2010) for the SGS stresses.

Another popular approach for the parametrization of the turbine-induced forces in the context of RANS consists of using the blade-element theory (Glauert 1935) to calculate the lift and drag forces and distribute them on the actuator disk. Here, this approach will be referred to as the 'actuator-disk model with rotation' (ADM-R). Typically, the blade-element theory is used to calculate both lift and drag forces from two-dimensional aerofoil characteristics, corrected for three-dimensional effects. Numerical simulations of actual wind turbines employing the ADM-R have been carried out by Sørensen and Kock (1995), Masson et al. (1997), Sørensen et al. (1998) in axisymmetric flow conditions, and by Ammara et al. (2002), 
Alinot and Masson (2002), Kasmi and Masson (2008) in ABL flow simulated using the RANS approach with a $\kappa-\varepsilon$ turbulence closure model. However, the ADM-R has not been tested in the context of LES.

In this study, we propose a novel LES framework that uses a tuning-free Lagrangian scaledependent dynamic model (Stoll and Porté-Agel 2006) for the SGS stress, together with an actuator-disk approach (ADM-R as well as ADM-NR) to model the turbine-induced forces. The framework and model formulations are presented in Sect. 2. The LES framework is then tested against wind-tunnel measurements of turbulence characteristics measured in the wake of a miniature wind turbine. The numerical and wind-tunnel experiments are described in Sect. 3, and the results are presented in Sect. 4. Summary is given in Sect. 5.

\section{Large-Eddy Simulation Framework}

LES solves the filtered incompressible Navier-Stokes equations, which can be written in rotational form as:

$$
\frac{\partial \widetilde{u}_{i}}{\partial x_{i}}=0
$$

and

$$
\frac{\partial \widetilde{u}_{i}}{\partial t}+\widetilde{u}_{j}\left(\frac{\partial \widetilde{u}_{i}}{\partial x_{j}}-\frac{\partial \widetilde{u}_{j}}{\partial x_{i}}\right)=-\frac{\partial \widetilde{p}^{*}}{\partial x_{i}}-\frac{\partial \tau_{i j}}{\partial x_{j}}+v \frac{\partial^{2} \widetilde{u}_{i}}{\partial x_{j}^{2}}-\frac{f_{i}}{\rho}+\delta_{i 1} F_{P},
$$

where $\widetilde{u}_{i}$ is the filtered velocity in the $i$-direction (with $i=1,2,3$ corresponding to the streamwise (x), spanwise (y) and wall-normal (z) directions, respectively), $\widetilde{p}^{*}=\widetilde{p} / \rho+\frac{1}{2} \widetilde{u}_{i} \widetilde{u}_{i}$ is the modified kinematic pressure, $\tau_{i j}=\widetilde{u_{i} u_{j}}-\widetilde{u}_{i} \widetilde{u}_{j}$ is the kinematic SGS stress, $v$ is the air kinematic viscosity, $f_{i}$ is an immersed forcing used to simulate effects of the turbine in the flow, $F_{P}$ is an imposed pressure gradient and $\rho$ is the constant air density (following the Boussinesq approximation). The rotational form of the equation is used for kinetic energy and mass conservation. This study focuses on the effect of boundary-layer turbulence on wind-turbine wakes in a neutrally-stratified wind-tunnel boundary-layer flow, and therefore Coriolis and buoyant effects are neglected. Next, we discuss the two parametrizations required in simulations of ABL flow with wind turbines: (a) the SGS stress model, and (b) the model for the turbine-induced forces.

\subsection{Lagrangian Scale-Dependent Dynamic SGS Stress Model}

The Smagorinsky model (Smagorinsky 1963) for the deviatoric part of the SGS stress is:

$$
\tau_{i j}-\frac{1}{3} \delta_{i j} \tau_{k k}=-2\left(C_{S} \Delta\right)^{2}|\widetilde{S}| \widetilde{S}_{i j},
$$

where $\widetilde{S}_{i j}=1 / 2\left(\partial \widetilde{u}_{i} / \partial x_{j}+\partial \widetilde{u}_{j} / \partial x_{i}\right)$ is the resolved strain-rate tensor, $\Delta$ is the spatial filter size and $C_{S}$ is the Smagorinsky coefficient. The model coefficient has been well established for isotropic turbulent flows (e.g., Smagorinsky 1963). However, a priori experimental studies (Porté-Agel et al. 2001a; Kleissl et al. 2003) as well as numerical simulations (Deadorff 1980; Porté-Agel et al. 2001b) have shown that the model coefficient should have a smaller value in the near-surface region of turbulent boundary layers in order to account for the reduction of the characteristic length scale of the turbulence associated with large shear and flow anisotropy found in that region of the flow. 
One systematic way to account for the spatial and temporal variability of the model coefficient is to use a Lagrangian scale-dependent dynamic model. The model computes the Smagorinsky coefficient $C_{S}$ at every point in the flow using the scale-dependent procedure developed by Porté-Agel et al. (2000) with the Lagrangian averaging introduced by Meneveau et al. (1996). Below, a brief summary of the procedure is given.

The dynamic model is based on the Germano identity (Germano et al. 1991)

$$
L_{i j}=\overline{\widetilde{u}}_{i} \widetilde{u}_{j}-\overline{\widetilde{u}}_{i} \overline{\tilde{u}}_{j}=T_{i j}-\bar{\tau}_{i j}
$$

where $L_{i j}$ is a resolvable turbulent stress tensor and $T_{i j}$ is the SGS stress at a test filter scale $\bar{\Delta}=\alpha \Delta$ (typically $\alpha=2$ ). The test filter SGS stress may be calculated using the eddy-viscosity model as

$$
T_{i j}-\frac{1}{3} \delta_{i j} T_{k k}=-2\left[C_{S}(\bar{\Delta}) \cdot \alpha \Delta\right]^{2}|\widetilde{\widetilde{S}}| \widetilde{\widetilde{S}}_{i j}
$$

Substituting Eqs. 3 and 5 into Eq. 4 results in the following system of equations describing the error associated with the use of the Smagorinsky model in the Germano identity:

$$
e_{i j}=L_{i j}-\frac{1}{3} \delta_{i j} L_{k k}-C_{S}^{2}(\Delta) M_{i j}
$$

where $M_{i j}=2 \Delta^{2}\left(\left|\widetilde{\widetilde{S} \mid \widetilde{S}_{i j}}-\alpha^{2} \beta\right| \widetilde{\widetilde{S}} \mid \widetilde{\widetilde{S}}_{i j}\right)$ and $\beta=C_{S}^{2}(\alpha \Delta) / C_{S}^{2}(\Delta)$ is the ratio of the model coefficient at the test filter scale and the filter scale. By means of least squares minimisation of the error given by Eq. 6, the optimal value of model coefficient $C_{S}^{2}$ (Ghosal et al. 1995) results in

$$
C_{S}^{2}(\Delta)=\frac{\left\langle L_{i j} M_{i j}\right\rangle}{\left\langle M_{i j} M_{i j}\right\rangle},
$$

where the brackets represent Lagrangian averaging.

Because a value of $\beta$ in Eq. 7 is required to close the model, a second test filter at a scale $\widehat{\Delta}=\alpha^{2} \Delta$ is adopted. Applying the Germano identity at that scale and minimising the error associated with one of the previously discussed averaging operators results in a second equation for $C_{S}^{2}(\Delta)$

$$
C_{S}^{2}(\Delta)=\frac{\left\langle Q_{i j} N_{i j}\right\rangle}{\left\langle N_{i j} N_{i j}\right\rangle},
$$

where $Q_{i j}=\widehat{\widetilde{u}_{i} \widetilde{u}_{j}}-\widehat{\widehat{u}}_{i} \widehat{\widetilde{u}}_{j}$ and $N_{i j}=2 \Delta^{2}\left(\widetilde{|\widetilde{S}| \widetilde{S}_{i j}}-\alpha^{4} \beta^{2} \mid \widehat{\widetilde{S}}_{\widehat{\widetilde{S}}_{i j}}\right)$. By equating Eqs. 7 and 8 , a polynomial equation of the unknown scale-dependence parameter $\beta$ can be derived to dynamically calculate $\beta$ (Porté-Agel et al. 2000). It is then substituted into Eq. 7 to calculate $C_{S}$. Further information on the polynomial equation and its solution as well as the Lagrangian averaging procedure has been reported in Porté-Agel et al. (2000) and Stoll and Porté-Agel (2006).

\subsection{Wind-Turbine Models}

To parametrise the turbine-induced forces, we use two versions of the actuator-disk models: ADM with rotation (ADM-R) and without rotation (ADM-NR). For both models, the actuator disk is denoted as the area swept by the blades, where the turbine-induced forces are parametrised and integrated over the spatial and temporal resolution used in LES. The standard actuator-disk model without rotation (ADM-NR) uses the one-dimensional momentum 
theory to construct the constantly loaded actuator disk with a thrust force uniformly distributed over the disk and acting in the streamwise direction (Jimenez et al. 2007, 2008). The thrust force on a cell is calculated as follows:

$$
F_{x}=\frac{\rho \tilde{u}_{0}^{2}}{2} A_{f} C_{T},
$$

where $\widetilde{u}_{0}$ is the unperturbed resolved streamwise velocity of the incident flow in the centre of the rotor disk, $A_{f}$ is the frontal area of cells within the rotor disk and $C_{T}$ is the thrust coefficient of the wind turbine.

In the ADM-R, the lift and drag forces acting on the turbine blades are parametrised using the blade-element theory, which has been previously used in the context of RANS simulations of flows through propellers (e.g., Phillips et al. 2009) and turbines (e.g., Ammara et al. 2002). In the blade-element theory, the turbine blades are divided into $N$ blade elements (see Fig. 1) that are assumed to behave aerodynamically as two-dimensional aerofoils. Based on momentum balance around the aerofoils, the aerodynamic forces are determined using the lift and drag characteristics of the aerofoil type as well as the local flow conditions. Note that for each blade element, the lift and drag forces are perpendicular and parallel, respectively, to the direction of the local relative velocity. Figure 1 shows a schematic of the various forces, velocities and angles for a cross-sectional blade element at radius $r$ in the $(x, \theta)$ plane of the cylindrical coordinate system, looking down from the blade tip. The axial and tangential velocities of the incident flow at blades are denoted as $V_{x}=V_{x}(r, \theta)$ and $V_{\theta}=V_{\theta}(r, \theta)$, respectively, in the inertial frame of reference. The local relative velocity with respect to the aerofoil element is defined as $\mathbf{V}_{\text {rel }}=\left(V_{x}, \Omega r-V_{\theta}\right)$, where $\Omega$ is the turbine angular velocity. The angle of attack is defined as

$$
\alpha=\phi-\gamma,
$$

where $\phi=\tan ^{-1}\left[V_{x} /\left(\Omega r-V_{\theta}\right)\right]$ is the angle between $V_{r e l}$ and the rotor plane and $\gamma$ is the local pitch angle. Lift and drag forces are calculated as

$$
(L, D)=\frac{\rho V_{r e l}^{2}}{2} c \Delta r\left(C_{L} \mathbf{e}_{\mathbf{L}}, C_{D} \mathbf{e}_{\mathbf{D}}\right),
$$

where $C_{L}=C_{L}\left(\alpha, R e_{c}\right)$ and $C_{D}=C_{D}\left(\alpha, R e_{c}\right)$ are the lift and drag coefficients obtained from tabulated aerofoil data, respectively, $R e_{c}$ is the Reynolds number based on the relative

Fig. 1 A cross-sectional aerofoil element

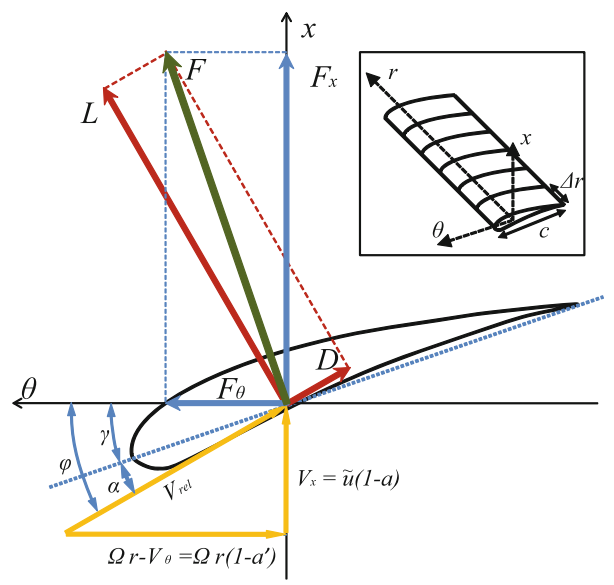


velocity and chord length, $\mathbf{e}_{\mathbf{L}}$ and $\mathbf{e}_{\mathbf{D}}$ are the direction of the unit vector for the lift and drag forces, respectively, $\Delta r$ is the radial length of the two-dimensional aerofoil element and $c$ is the chord length. Because it is assumed that the turbine has no radial action on the flow, the turbine-induced forces acting on each blade element are expressed as

$$
\mathbf{F}=\frac{\rho V_{r e l}^{2}}{2} c \Delta r\left(C_{L} \mathbf{e}_{\mathbf{L}}+C_{D} \mathbf{e}_{\mathbf{D}}\right) .
$$

Unlike the ADM-NR, the ADM-R considers the effect of the turbine-induced flow rotation as well as the non-uniform force distribution. To determine the forces acting on the rotor disk, we consider an annular area of differential size $d A=2 \pi r d r$. The resulting force per unit rotor area is given by

$$
f_{2 D}=\frac{d \mathbf{F}}{d A}=\frac{\rho V_{r e l}^{2}}{2} \frac{B c}{2 \pi r}\left(C_{L} \mathbf{e}_{\mathbf{L}}+C_{D} \mathbf{e}_{\mathbf{D}}\right),
$$

where $B$ is the number of blades.

The blade-element theory requires to specify the axial and tangential components of the incident flow velocity at blades. In order to achieve that, we assume $V_{x}=\widetilde{u}(1-a)$ and $V_{\theta}=\Omega r a^{\prime}$ (as shown in Fig. 1), where $\tilde{u}$ is the unperturbed instantaneous streamwise velocity, and $a$ and $a^{\prime}$ are the induction factors in the streamwise and tangential directions, respectively. Then, we use the blade-element momentum (BEM) theory (Manwell et al. 2002) to calculate the incident flow velocities $\left(V_{x}, V_{\theta}\right)$, the induction factors $\left(a, a^{\prime}\right)$, and the lift and drag coefficients $\left(C_{L}, C_{D}\right)$. The BEM theory originates from two different theories based on the momentum balance of the flow. The first one is the above-mentioned blade-element theory, which parametrizes the lift and drag forces at each blade section (element) based on the momentum balance around the aerofoil; the second is the one-dimensional momentum theory, which parametrizes the thrust and torque by applying the conservation of linear and angular momentum equations, respectively, to the control volume of a rotating annular stream tube through the wind turbine. The combination of these two theories gives a series of BEM equations that can be solved by numerical iteration. Details of the BEM procedure are given by Manwell et al. (2002).

In this study, the thrust coefficient $C_{T}$, required to calculate the thrust force in the ADMNR (Eq. 9), is estimated based on the drag $(D)$ and lift $(L)$ forces obtained with the BEM theory. Because the ADM-NR considers an overall thrust force $(T)$ and ignores the effect of the turbine-induced flow rotation, a constant thrust coefficient applied on the whole rotor disk is calculated using

$$
C_{T}=\frac{T}{0.5 \widetilde{u}_{0}^{2} A_{D}},
$$

where $T=B \int_{0}^{R}\left(\frac{d L}{d r} \cos \phi+\frac{d D}{d r} \sin \phi\right) d r$ is the total thrust over the rotor area, $A_{D}=\pi R^{2}$ is the area of the rotor disk, and $R$ is the rotor radius.

In both wind-turbine models, a parametrization is needed for the forces induced by the nacelle on the turbulent flow. The nacelle is described as a permeable disk area $A_{\text {nac }}$ with a delimited radius $r_{n a c}$. The nacelle-induced drag force acting upon the incoming flow is parametrized using a formulation similar to the ADM-NR:

$$
F_{x}=\frac{\rho \widetilde{u}_{0}^{2}}{2} A_{n a c} C_{D, n a c}, \quad r \leq r_{n a c},
$$

where $C_{D, n a c}$ is the drag coefficient of the nacelle. The same approach is also applied to model the effect of the turbine tower with a drag coefficient of the tower $C_{D \text {,tower }}$. 
In both ADM-NR and ADM-R, the parametrized forces are distributed smoothly to avoid singular behaviour and numerical instability. In practice the aerodynamic blade forces are distributed in a three-dimensional Gaussian manner by taking the convolution of the computed local load, F, and a regularisation kernel $\eta_{\epsilon}$ as (Mikkelsen 2003)

$$
\mathbf{f}=\frac{1}{\Delta v}\left(\mathbf{F} \otimes \eta_{\epsilon}\right)
$$

where

$$
\eta_{\epsilon}=\frac{1}{\epsilon^{3} \pi^{3 / 2}} \exp \left(-\frac{d^{2}}{\epsilon^{2}}\right),
$$

$\Delta v$ is the volume of a grid cell, $\epsilon$ is a constant parameter that adjusts the distribution of the regularised load, and $d$ is the distance between grid points and blade elements. Following Mikkelsen (2003), here we have chosen a value of $\epsilon$ equal to the grid-cell size in the streamwise direction.

\section{Numerical Experiments}

Large-eddy simulations are performed of a neutrally-stratified boundary-layer flow through an isolated wind turbine over a flat homogeneous surface. An actuator-disk model for the turbine-induced surface forces is implemented in the LES of a turbulent boundary layer driven by a constant streamwise pressure gradient. A modified version of the LES code described by Albertson and Parlange (1999), Porté-Agel et al. (2000), Porté-Agel (2004) and Stoll and Porté-Agel (2006) is used. The turbine-induced forces are modelled using the abovementioned actuator-disk models (ADM-R and ADM-NR). The main features of the code and a description of the case study are briefly summarised below.

The computational domain has a height $L_{z}=0.460 \mathrm{~m}$, corresponding to the wind-tunnel boundary-layer depth $H$ reported by Chamorro and Porté-Agel (2010). The horizontal computational domain spans a distance $L_{x}=28.8 d=2 \pi H$ in the streamwise direction and $L_{y}=4.8 d=\pi H / 3$ in the spanwise direction, where $d=0.150 \mathrm{~m}$ denotes the turbine diameter. As shown in Fig. 2, the wind turbine has a hub height $H_{h u b}=0.125 \mathrm{~m}$ and it is placed in the middle of the computational domain at a distance of six rotor diameters from the upwind boundary. The turbine rotor is roughly within the lowest third of the turbulent boundary layer. The bottom tip of the turbine is set to a height of 0.67 times the turbine radius, which is similar to that found in large turbines $(\geq 2 \mathrm{MW})$. The domain is uniformly divided into $N_{x} \times N_{y} \times N_{z}$ grid points with a spatial resolution of $\Delta_{x} \times \Delta_{y} \times \Delta_{z}$ in the streamwise, spanwise and wall-normal directions, respectively. The grid is staggered in the
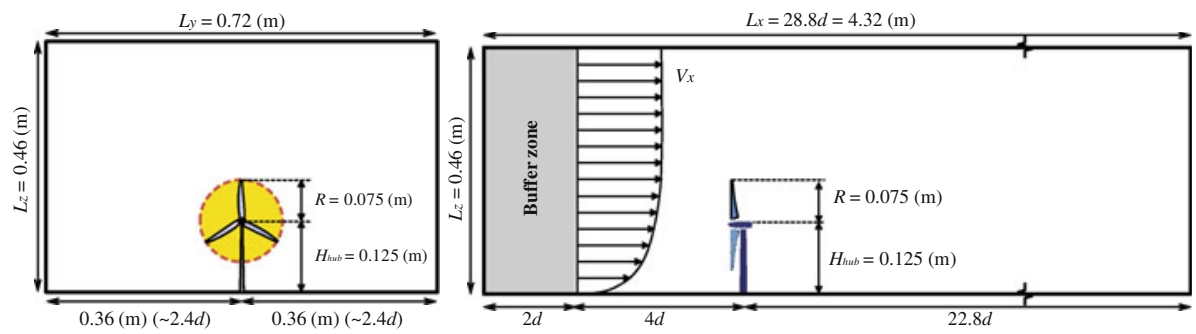

Fig. 2 Schematic of the simulation domain. Front view (left) and side view (right) 
Table 1 Numerical set-up for homogeneous neutral ABL simulations at different grid resolutions

\begin{tabular}{lcccccccc}
\hline Case & $N_{x}$ & $N_{y}$ & $N_{z}$ & $L_{x}(\mathrm{~m})$ & $L_{y}(\mathrm{~m})$ & $L_{z}(\mathrm{~m})$ & $N_{t, y}$ & $N_{t, z}$ \\
\hline ADM-R1 & 288 & 48 & 64 & 4.320 & 0.720 & 0.460 & 11 & 20 \\
ADM-R2 & 192 & 32 & 42 & 4.320 & 0.720 & 0.460 & 7 & 13 \\
ADM-R3 & 120 & 20 & 28 & 4.320 & 0.720 & 0.460 & 5 & 8 \\
ADM-R4 & 96 & 32 & 21 & 4.320 & 0.720 & 0.460 & 7 & 7 \\
\hline
\end{tabular}

vertical direction with the first level of computation for the vertical velocity $\widetilde{w}$ at a height of $\Delta_{z}=L_{z} /\left(N_{z}-1\right)$ and the first level of $\widetilde{u}, \widetilde{v}$ and $\widetilde{p}^{*}$ at $\Delta_{z} / 2$. Four different spatial resolutions (see Table 1) were used to test the resolution sensitivity of the simulation results. In Table 1, $N_{t, y}$ and $N_{t, z}$ denote the number of grid points covering the rotor diameter in spanwise and vertical directions, respectively.

The numerical scheme is based on a mixed pseudospectral finite-difference method, i.e., spatial derivatives are computed using pseudospectral representation in the horizontal directions and finite differences in the vertical direction. As a result, the lateral boundary conditions are periodic. The top boundary condition is a fixed stress-free lid, i.e., $d \widetilde{u} / d z=d \widetilde{v} / d z=$ $\widetilde{w}=0$. The bottom boundary condition consists of using similarity theory (Monin-Obukhov similarity) to calculate the instantaneous (filtered) surface shear stress as a function of the velocity field at the lowest vertical grid point $\Delta z / 2$. The code is fully dealiased using the $3 / 2$ rule (Canuto et al. 1988) and time advancement is done using a second-order accurate Adams-Bashforth scheme. In the SGS model formulations the filter width is calculated with the standard formulation $\Delta=\left(\Delta_{x} \Delta_{y} \Delta_{z}\right)^{1 / 3}$. Test filtering at scales $2 \Delta$ and $4 \Delta$, needed for the scale-dependent procedure, is done with a sharp spectral cut-off filter. The model coefficients are computed every three timesteps to increase computational efficiency.

To validate the LES code, the simulation results are compared with high-resolution velocity measurements collected in the wake of a three-blade miniature wind turbine placed in the St. Anthony Falls Laboratory atmospheric boundary-layer wind tunnel. The experimental set-up is given by Chamorro and Porté-Agel (2010). In this experiment, the miniature turbine consists of a three-blade GWS/EP- $6030 \times 3$ rotor attached to a small DC generator motor. The hub height is $0.125 \mathrm{~m}$ and the rotor diameter is $0.150 \mathrm{~m}$. The motor has a cylindrical shape with a diameter $d_{m}=0.015 \mathrm{~m}$ and a length $l_{m}=0.03 \mathrm{~m}$. The turbine tower is also a cylindrical shape with a diameter $d_{m}=0.005 \mathrm{~m}$ and a length $l_{m}=0.118 \mathrm{~m}$. The drag coefficient of bluff bodies of shape similar to typical cylinders varies between 0.8 and 1.2 (Schetz and Fuhs 1996; Kasmi and Masson 2008), depending on the their geometry and fineness ratio. For the case of the cylindrical nacelle and the turbine tower considered here, the drag coefficients of the nacelle $\left(C_{D, \text { nac }}\right)$ and the turbine tower $\left(C_{D, \text { tower }}\right)$ are set to 0.85 and 1.2 , respectively. In our simulations, the blade section is assumed to be a flat plate from root to tip. We adopt the data of lift and drag coefficients versus the angle of attack, obtained from experimental results Sunada et al. (1997), for the appropriate flat plate at different Reynold numbers.

The radial variation of the chord length and pitch angle is presented in Table 2. Highresolution velocity measurements were collected with hot-wire anemometry in the wake of the miniature turbine in the middle of the domain as well as a cross-sectional plane downstream of $x / d=5$. More details on the hot-wire anemometry system and calibration can be found in Chamorro and Porté-Agel $(2009,2010)$. The boundary-layer flow developed at the downwind end of the $16 \mathrm{~m} \times 1.7 \mathrm{~m} \times 1.7 \mathrm{~m}$ test section of the tunnel is characterised by a free stream velocity of $2.8 \mathrm{~m} \mathrm{~s}^{-1}$ and a boundary-layer depth of $0.46 \mathrm{~m}$. The inflow velocity at the 
Table 2 Radial variation of the pitch angle and chord length for the miniature turbine

\begin{tabular}{llllllllll}
\hline Radius & $(\mathrm{m})$ & 0.01 & 0.02 & 0.03 & 0.04 & 0.05 & 0.06 & 0.07 & 0.075 \\
\hline Pitch & $($ degree $)$ & 20.50 & 20.90 & 19.83 & 16.91 & 13.19 & 10.67 & 9.12 & 6.66 \\
Chord & $(\mathrm{m})$ & 0.0139 & 0.0147 & 0.0147 & 0.0141 & 0.0131 & 0.0118 & 0.0102 & 0.0060 \\
\hline
\end{tabular}

hub height (i.e., $\bar{u}_{h u b}$ ) is $2.2 \mathrm{~m} \mathrm{~s}^{-1}$. The value of the friction velocity and surface roughness are $0.102 \mathrm{~m} \mathrm{~s}^{-1}$ and $0.03 \mathrm{~mm}$, respectively. The average turbine angular velocity is $1120 \mathrm{rpm}$, which corresponds to a tip speed ratio of approximately 4 based on the inflow velocity at the hub height. The tip speed ratio was adjusted to match that of field-scale turbines, usually between 3 and 8 (Hau 2000). A similar range of the tip speed ratio, between 3 and 10 (Vermeer et al. 2003), was also chosen in a number of wind-tunnel studies of wind-turbine wakes, both for single turbines and wind farms.

To avoid the turbine-induced wake flow to affect the flow upwind of the turbine due to the periodic boundary conditions, a buffer zone upstream of the wind turbine (Fig. 2) is employed to adjust the flow from the very-far-wake downwind condition to that of an undisturbed boundary-layer inflow condition. This inflow condition is obtained from a separate simulation of the boundary-layer flow corresponding to the upwind of the wind turbine in the wind-tunnel experiment of Chamorro and Porté-Agel (2010). The use of a similar buffer region to impose the inflow boundary condition while maintaining the accuracy of pseudospectral LES codes has been successful in former studies of turbulent transport in urban street canopies (Tseng et al. 2006) as well as over a steep hill topography (Wan and Porté-Agel 2010). In this study, all numerical simulations were run for a period of time long enough to guarantee quasi-steady flow conditions and statistical convergence of the results presented in the next section.

\section{Results}

In this section, numerical simulation results obtained with LES coupled with the two actuator-disk models (ADM-NR and ADM-R) are compared with the high-resolution windtunnel measurements. In particular, we focus on the spatial distribution of three key turbulence statistics used to characterise wind-turbine wakes: the time-averaged streamwise velocity $\bar{u}$, the streamwise turbulence intensity $\sigma_{u} / \bar{u}_{h u b}$, and the kinematic shear stress $-\overline{u^{\prime} w^{\prime}}$. The overbar denotes a temporal average. The experimental data were collected at $x / d=-1,1,2, \ldots, 9,10,12, \ldots, 18,20$ in the middle of the domain as well as $y / d=-0.7,-0.6, \ldots, 0.6,0.7$ in a cross-section downstream $(x / d=5)$ of the turbine.

Figure 3 displays contours of the time-averaged streamwise velocity obtained from the wind-tunnel experiment (Fig. 3a) and simulations with both ADM-R (Fig. 3b) as well as ADM-NR (Fig. 3c) on a vertical plane perpendicular to the turbine. Furthermore, to facilitate the quantitative comparison of the results, vertical profiles of the measured and simulated time-averaged streamwise velocity are shown in Fig. 4 for selected downwind locations $(x / d=2,3,5,7,10,14,20)$, together with the incoming flow velocity profile. From the measurements, there is clear evidence of the effect of the turbine extracting momentum from the incoming flow and producing a wake (region of reduced velocity) immediately downwind. As expected the velocity deficit (reduction with respect to the incoming flow) is largest near the turbine and it becomes smaller as the wake expands and entrains surrounding air. 

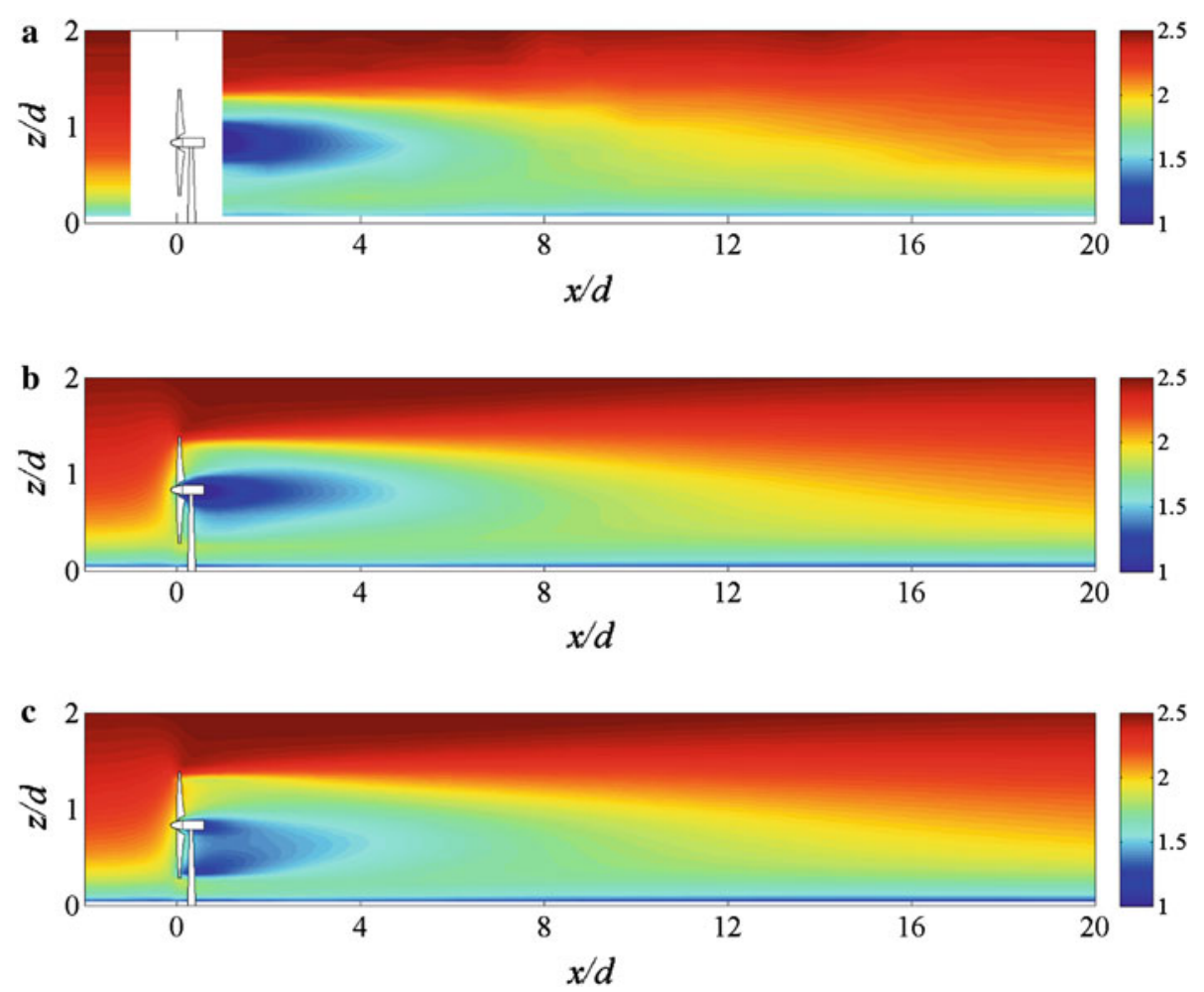

Fig. 3 Contours of the time-averaged streamwise velocity $\bar{u}\left(\mathrm{~m} \mathrm{~s}^{-1}\right)$ in the middle vertical plane perpendicular to the turbine: a wind-tunnel measurements, b ADM-R, c ADM-NR

Nonetheless, the effect of the wake is still noticeable even in the far wake, at distances as large as $x / d=20$.

Due to the non-uniform (logarithmic) mean velocity profile of the incoming boundarylayer flow, we find a non-axisymmetric distribution of the mean velocity profile and, consequently, of the mean shear in the turbine wake. In particular, as also reported by Chamorro and Porté-Agel (2009), the strongest shear (and associated turbulence kinetic energy production) is found at the level of the top tip. This result contrasts with the axisymmetry of the turbulence statistics reported by previous studies in the case of wakes of turbines placed in free-stream flows (e.g., Medici and Alfredsson 2006; Troldborg et al. 2007), and demonstrates the substantial influence of the incoming flow on the structure and dynamics of wind-turbine wakes.

From Figs. 3 and 4, LES with the scale-dependent dynamic SGS model and the ADM-R turbine model yields mean velocity profiles that are in good agreement with the measurements everywhere in the turbine wake (near wake as well as far wake). The ADM-NR is able to capture the velocity distribution in the far-wake region $(x / d>5)$, but it clearly overpredicts the velocity in the centre of the wake in the near-wake regions $(x / d<5)$. This failure of the ADM-NR to reproduce the velocity magnitude in the near-wake region can be attributed to the limitations of two important assumptions made in the ADM-NR (but not in the ADM-R): (a) the effect of turbine-induced rotation is ignored, and (b) the force is uniformly distributed 

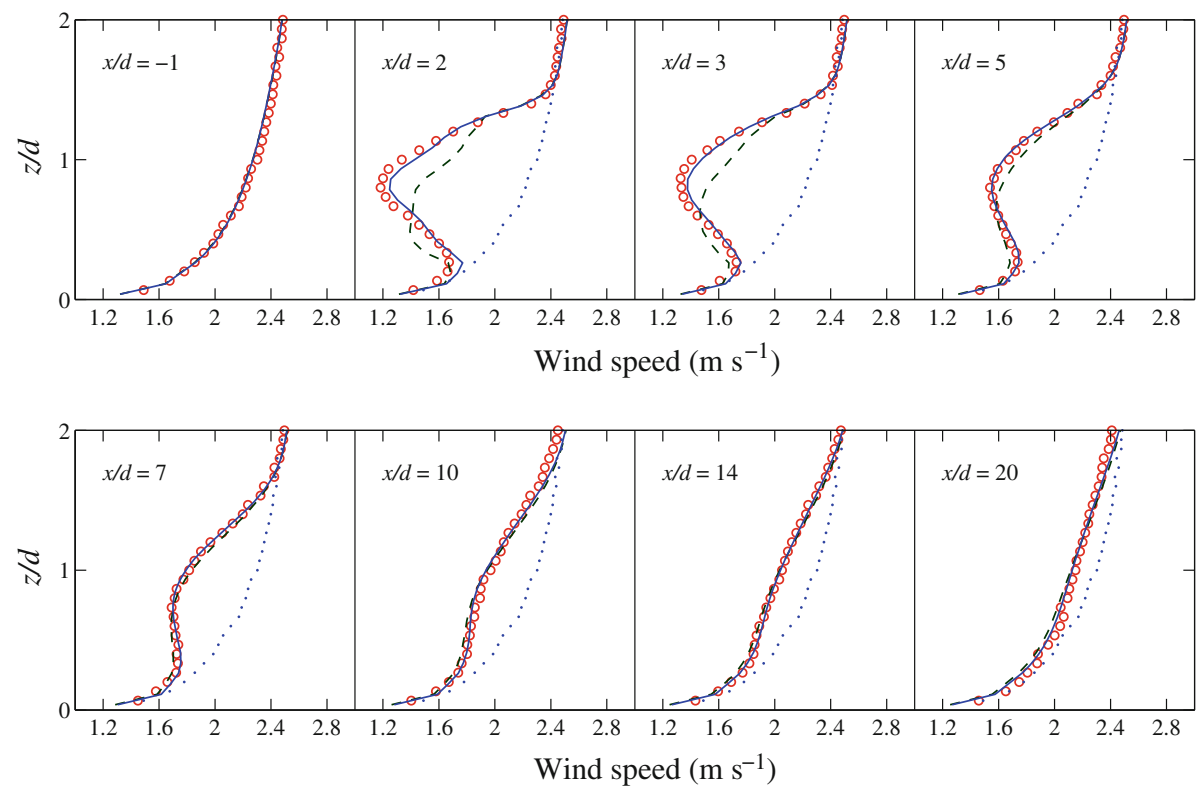

Fig. 4 Comparison of vertical profiles of the time-averaged streamwise velocity $\bar{u}\left(\mathrm{~m} \mathrm{~s}^{-1}\right)$ : wind-tunnel measurements (open circle), ADM-R (solid line) and ADM-NR (dashed line). The dotted line represents the inflow profile

over the rotor disk, thus ignoring the radial variation of the force. These two assumptions are in contrast with simulation results using the non-uniform force distribution reported by Sørensen and Shen (2002).

Contours of the average velocity at the lateral cross-section and downwind location $x / d=$ 5 are shown in Fig. 5. The results show the non-axisymmetry (with respect to the turbine axis) of the velocity distribution due to the non-uniformity of the incoming flow and the presence of the surface. Simulation results obtained from both ADM-NR and ADM-R are in acceptable agreement with the measurements, with only a small difference in the magnitude of the velocity for both models. This is consistent with the idea that, as mentioned above, both models give good mean velocity predictions in the far-wake region. The ADM-R is better able to predict the location of the elevated velocity minimum near the hub height at $x / d=5$. Both mean resolved velocity patterns are in acceptable agreement with the experimental measurements.

Figure 6 shows contours of the streamwise turbulence intensity $\sigma_{u} / \bar{u}_{h u b}$ obtained from the wind-tunnel measurements (Fig. 6a) and simulations (resolved part) using LES with ADMR (Fig. 6b) and ADN-NR (Fig. 6c) on a vertical plane perpendicular to the turbine. Again, vertical profiles of measured and simulated turbulence intensities at selected downwind locations $(x / d=2,3,5,7,10,14,20)$ are presented in Fig. 7 , together with the incoming flow turbulence intensity profile. As reported by Chamorro and Porté-Agel (2009), the measurements show a strong enhancement of the turbulence intensity (compared with the relatively low turbulence levels in the incoming flow) at the level of the top tip. Here, we find the maximum turbulence intensity at that level and at a normalised distance of approximately $3<x / d<5$. It is important to point out that this is within the typical range of distances between adjacent wind turbines in wind farms and, therefore, it should be considered when calculating wind loads on the turbines. The turbulence intensity distribution and, in partic- 

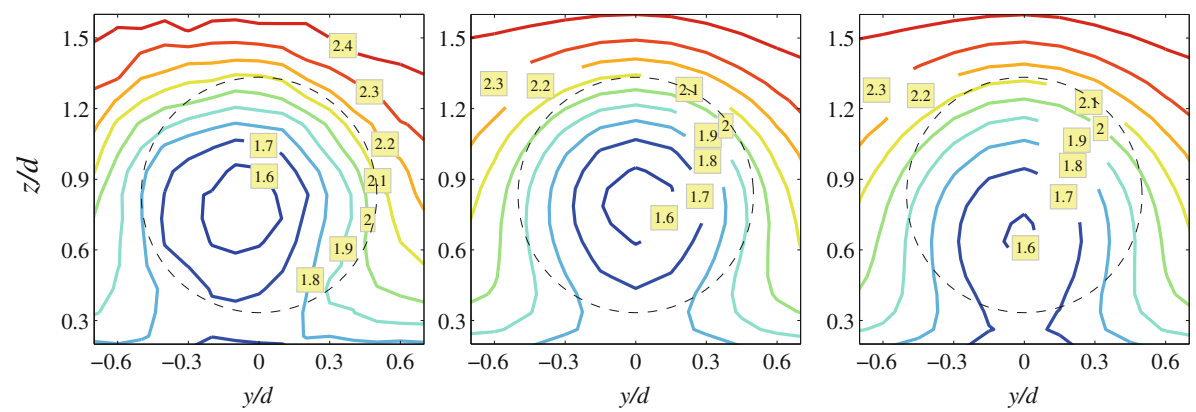

Fig. 5 Contours of the time-averaged streamwise velocity $\bar{u}\left(\mathrm{~m} \mathrm{~s}^{-1}\right)$ at the lateral cross section and downstream $x / d=5$ obtained from the wind-tunnel measurements (left), ADM-R (centre) and ADM-NR (right). The dashed line represents the turbine region
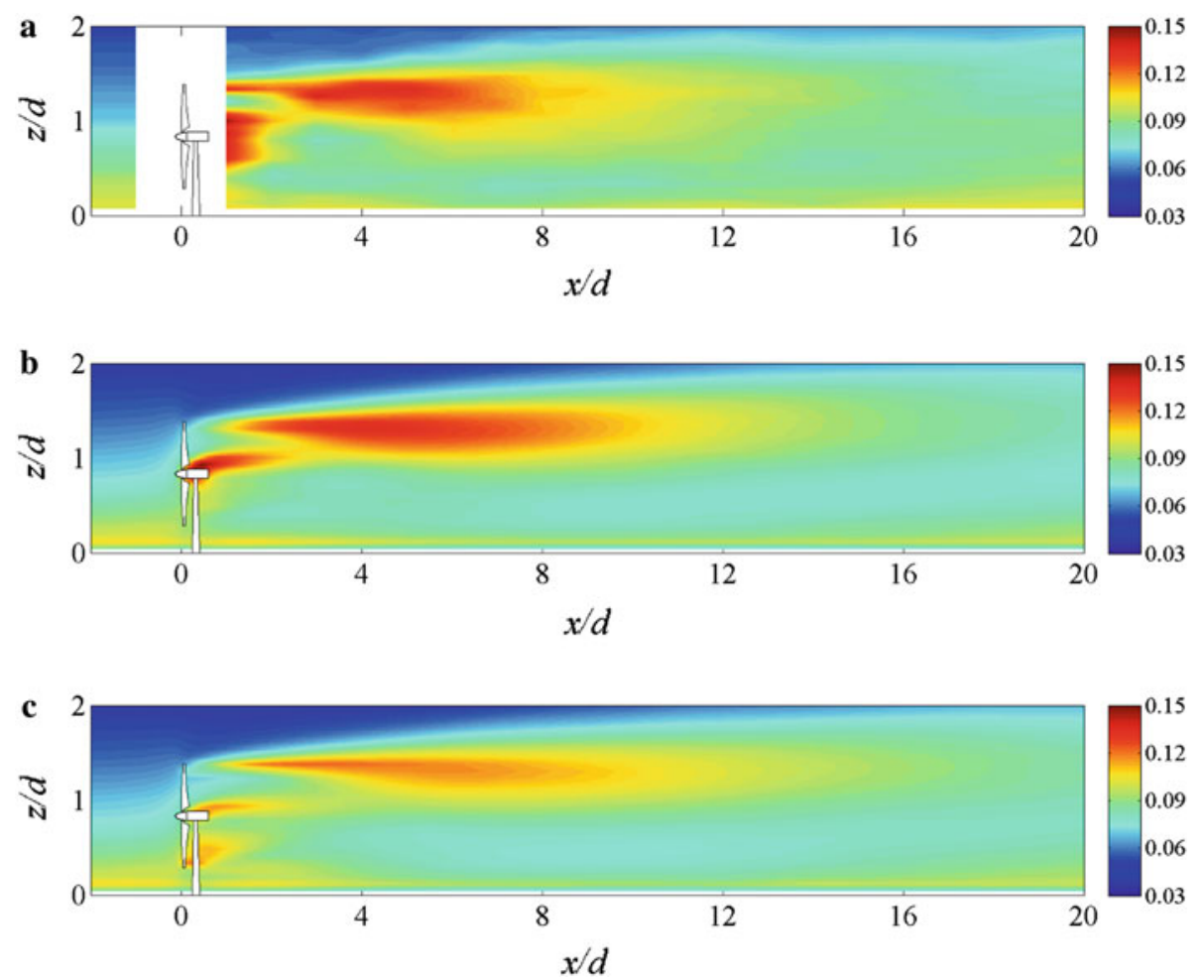

Fig. 6 Contours of the streamwise turbulence intensity $\sigma_{u} / \bar{u}_{h u b}$ in the middle vertical plane perpendicular to the turbine: a wind-tunnel measurements, b ADM-R, c ADM-NR

ular, the maximum enhancement of turbulence intensity occurs at the top-tip level can be explained considering the non-axisymmetric distribution of velocity profiles (Figs. 3, 4) and the fact that the mean shear and associated turbulence kinetic energy production are maximum at the top-tip height. The simulations with both wind-turbine models yield similar qualitative trends in the turbulence intensity distribution and the location of the maximum value. However, both models differ substantially in their ability to capture the magnitude of the turbulence intensity. In particular, the turbulence intensity magnitude obtained with LES and 

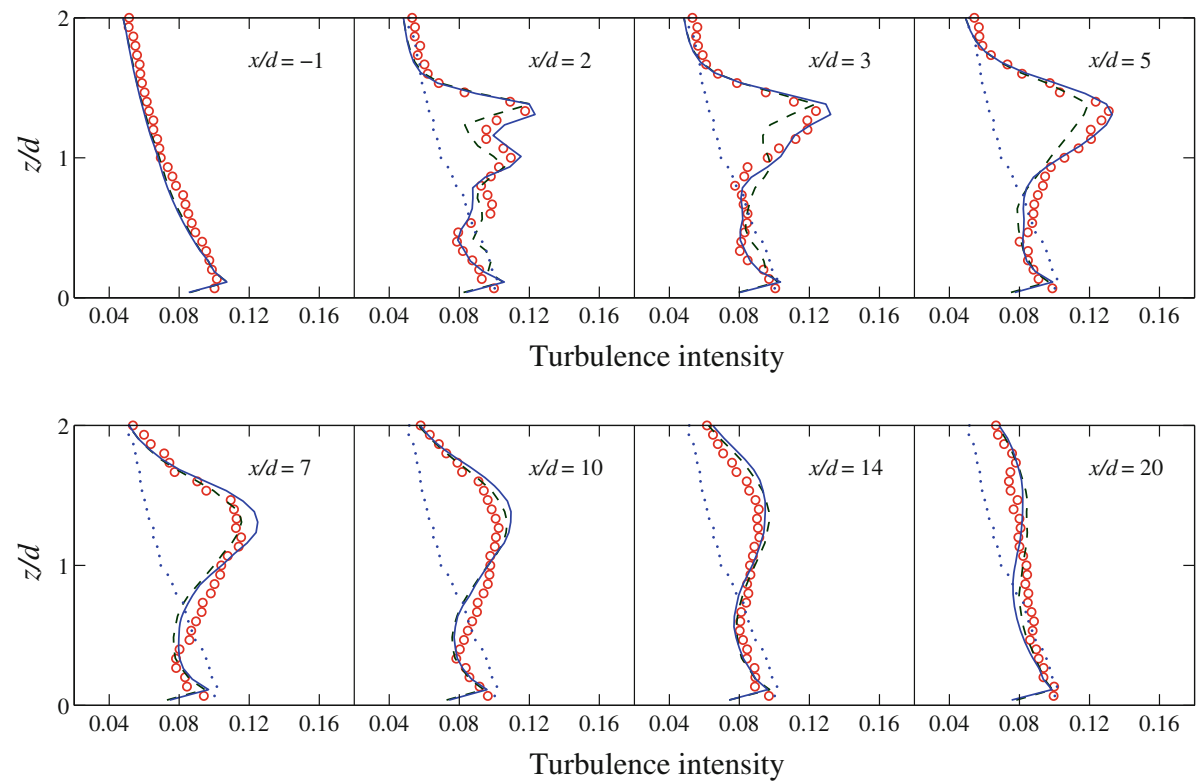

Fig. 7 Comparison of vertical profiles of the streamwise turbulence intensity $\sigma_{u} / \bar{u}_{h u b}$ : wind-tunnel measurements (open circle), ADM-R (solid line) and ADM-NR (dashed line). The dotted line represents the inflow profile

the ADM-R is good agreement with the wind-tunnel measurements. The ADM-NR clearly underestimates the wake turbulence intensity at the downstream positions of $x / d=2,3,5$ and 7 , and therefore its maximum value. However, like in the case of the mean velocity, both models give a good prediction in the far-wake region, particularly after 10 rotor diameters.

Figure 8 shows contour plots of the measured and simulated normalised turbulence intensity at the spanwise cross section corresponding to the downwind distance $x / d=5$. Like in the case of the average velocity, the turbulence intensity from both measurements and simulations show a clear non-axisymmetric distribution, with an inverse U-shape of the contour lines and a maximum turbulence intensity at the top-tip height. As explained above, this is the result of a non-uniform incoming flow and the presence of the surface. Consistent with the vertical profiles, for a given downwind distance, the maximum turbulence intensity is found at the top-tip height. Compared with the wind-tunnel experimental result, the ADM-R is much better to predict the distribution and magnitude of the turbulence intensity than the ADM-NR.

Figure 9 shows contour plots of kinematic shear stress $-\overline{u^{\prime} w^{\prime}}$ obtained from the experiment and the simulations on a vertical plane perpendicular to the turbine. The results show the turbine introduces kinematic stresses that are locally much larger in magnitude than the stresses in the incoming velocity field. In the near-wake region, up to a distance of about $x / d=5$, there is a region above the turbine hub and near the top-tip with large positive stress, and a lower region (below the hub height) with negative stress (Fig. 10). Like in the case of the turbulence intensity, the magnitude of the positive stress is largest at the top-tip height. Again, this is due to the high levels turbulence production associated with the strong shear at that level. As the turbine wake develops with downwind distance, the relative magnitude of the kinematic stress between the upper and lower parts of the near-wake region becomes 

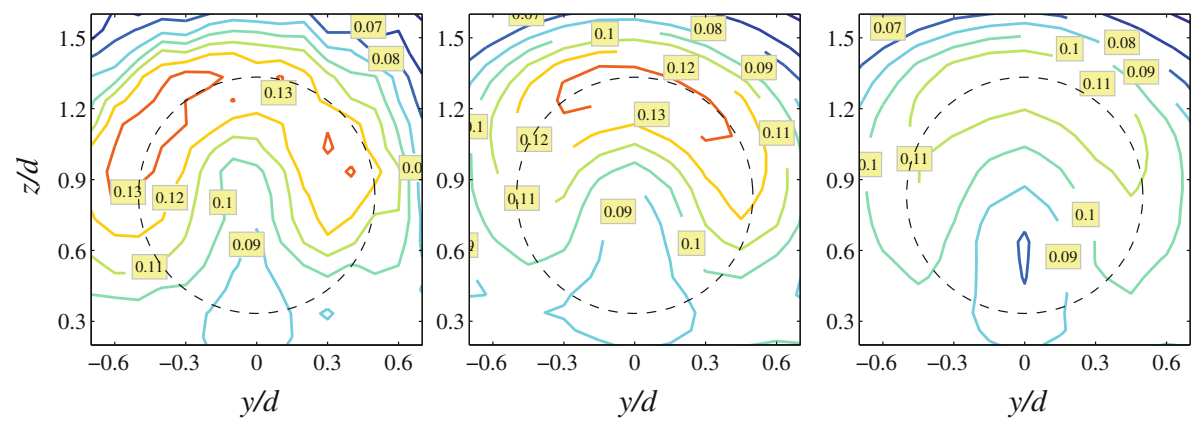

Fig. 8 Contours of the streamwise turbulence intensity $\sigma_{u} / \bar{u}_{h u b}$ at the lateral cross-section and downstream $x / d=5$ from the wind-tunnel measurements (left), ADM-R (centre) and ADM-NR (right). The dashed line represents the turbine region
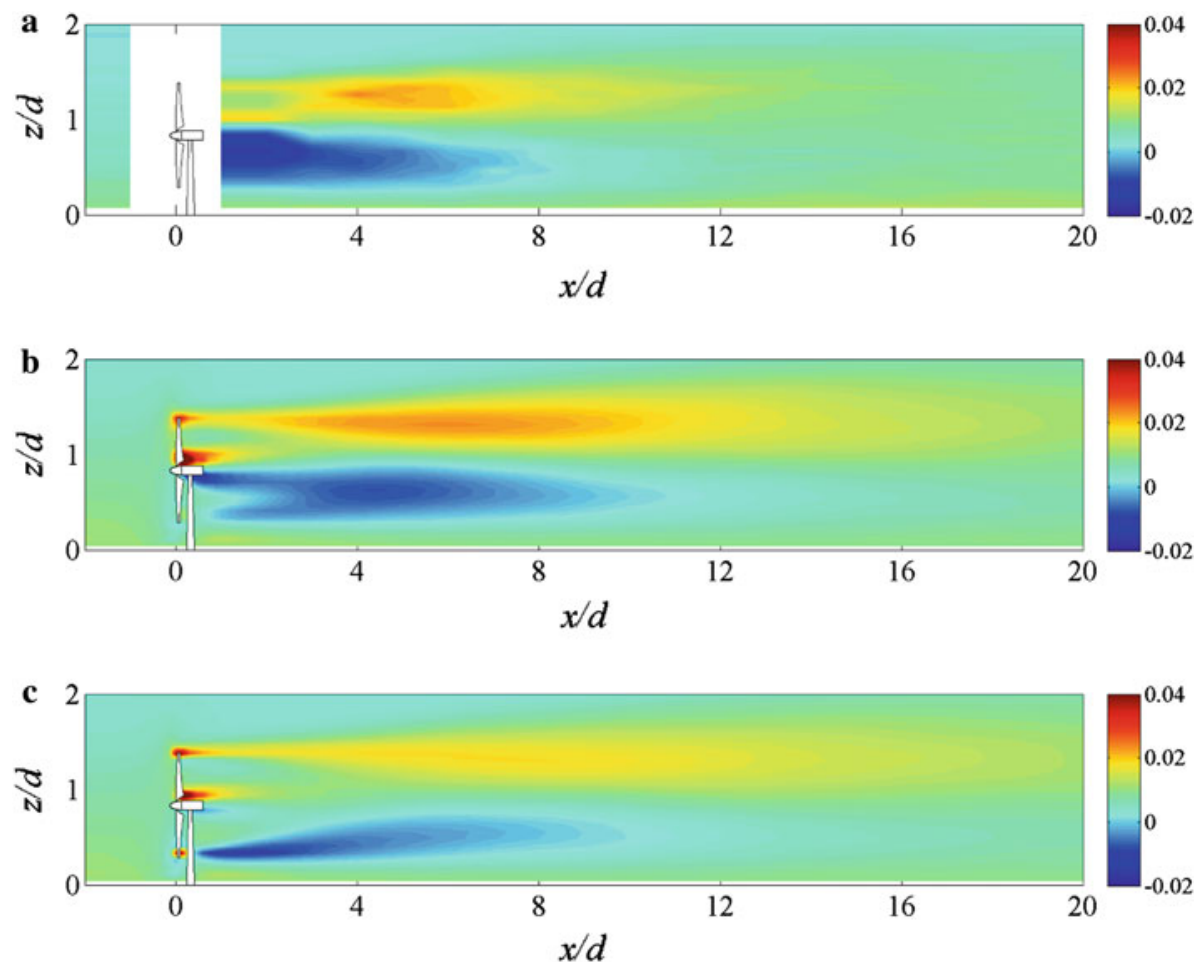

Fig. 9 Contours of the kinematic shear stress $-\overline{u^{\prime} w^{\prime}}\left(\mathrm{m}^{2} \mathrm{~s}^{-2}\right)$ in the middle vertical plane perpendicular to the turbine: a wind-tunnel measurements, b ADM-R, c ADM-NR

smaller and affects a larger cross-sectional area. Also similar to the other turbulence statistics (mean velocity and turbulence intensity), the change in kinematic stress with respect to the incoming flow is not negligible until the very far-wake region at a distance of $x / d=20$. Regarding the performance of the simulations, the ADM-R still shows a better prediction in the near-wake region compared with the ADM-NR. However, the ADM-R appears to overestimate the maximum magnitude of the turbulent stress at the top-tip height. The ADM-NR underestimates the magnitude of the shear stress in the near-wake region (at $x / d=2,3$ and 

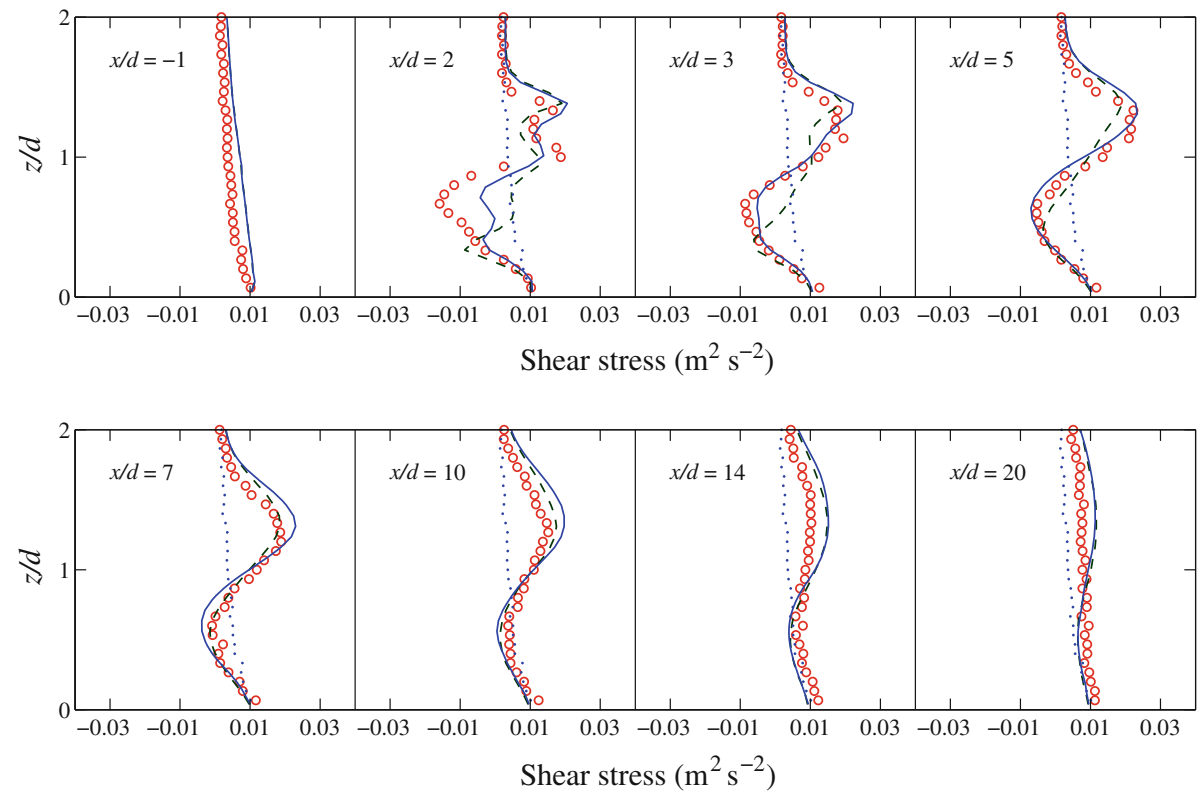

Fig. 10 Comparison of vertical profiles of the kinematic shear stress $-\overline{u^{\prime} w^{\prime}}\left(\mathrm{m}^{2} \mathrm{~s}^{-2}\right)$ : wind-tunnel measurements (open circle), ADM-R (solid line) and ADM-NR (dash line). The dotted line represents the inflow profile

5) in both upper and lower parts of the wake. In the very far wake region, at $x / d>10$, the simulated shear stress profiles from the two models are very similar and show a reasonable agreement (with a slight overestimation near the top-tip level) with the measurements.

In order to demonstrate the non-symmetrical distribution of the turbulent stress with respect to the $y=0$ plane, Fig. 11 presents the contour plots of the measured and simulated kinematic stress in a cross-sectional plane at the downwind position $x / d=5$. The wind-tunnel experimental data shows that, at that distance, the largest positive stress is located in the first quadrant (above the hub height and $y>0$ ), while the largest negative stress is found in the third quadrant (below the hub height and $y<0$ ). This tilting of the turbulent stress distribution can be attributed to the rotation of the wake. Simulation results obtained using the ADM-R show a similar tilting behaviour in the kinematic shear stress distribution, although the amount of tilting is underestimated compared with the measurements. This could also explain in part the difficulties of the model to capture the magnitude of the stress at the $y=0$ plane. The ADM-NR completely fails to predict the tilting behaviour of the kinematic stress due to the fact that this model does not allow for rotation of the wake.

As mentioned above, the ADM-R has two important advantages with respect to the ADMNR: it accounts for the non-uniform distribution of the thrust force, and it also considers tangential forces. To isolate the improvements caused by each one, a test simulation was preformed using a modified ADM-R that only considers the non-uniform distribution of the thrust force over the rotor disk, but neglects the tangential forces responsible for wake rotation. The simulation results obtained from both the ADM-R and the modified ADM-R (not shown here) are found to be quite similar, which indicates that accounting for the non-uniform distribution of the thrust force is responsible for most of the improvement observed in the ADM-R with respect to the ADM-NR. 

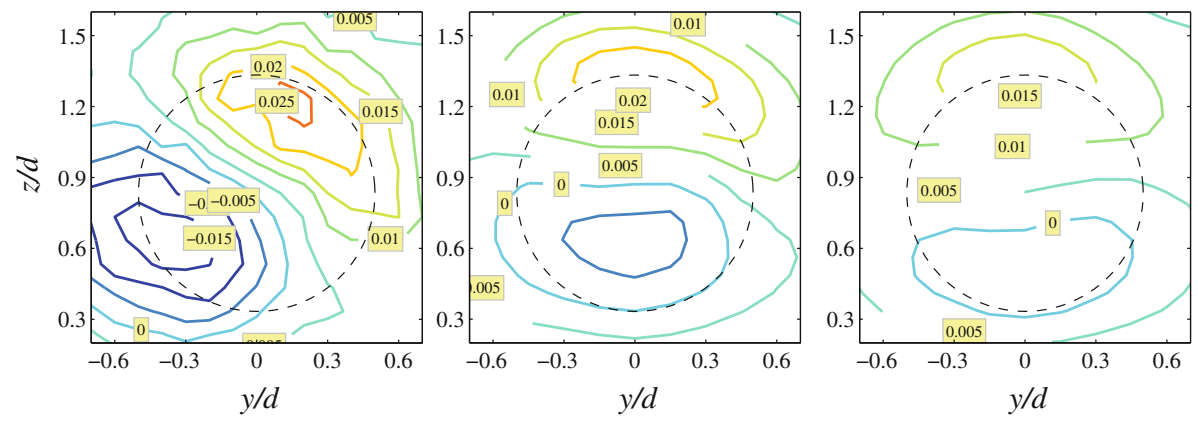

Fig. 11 Contours of the kinematic shear stress $-\overline{u^{\prime} w^{\prime}}\left(\mathrm{m}^{2} \mathrm{~s}^{-2}\right)$ at the lateral cross section and downstream $x / d=5$ from the wind-tunnel measurements (left), ADM-R (centre) and ADM-NR (right). The dashed line represents the turbine region
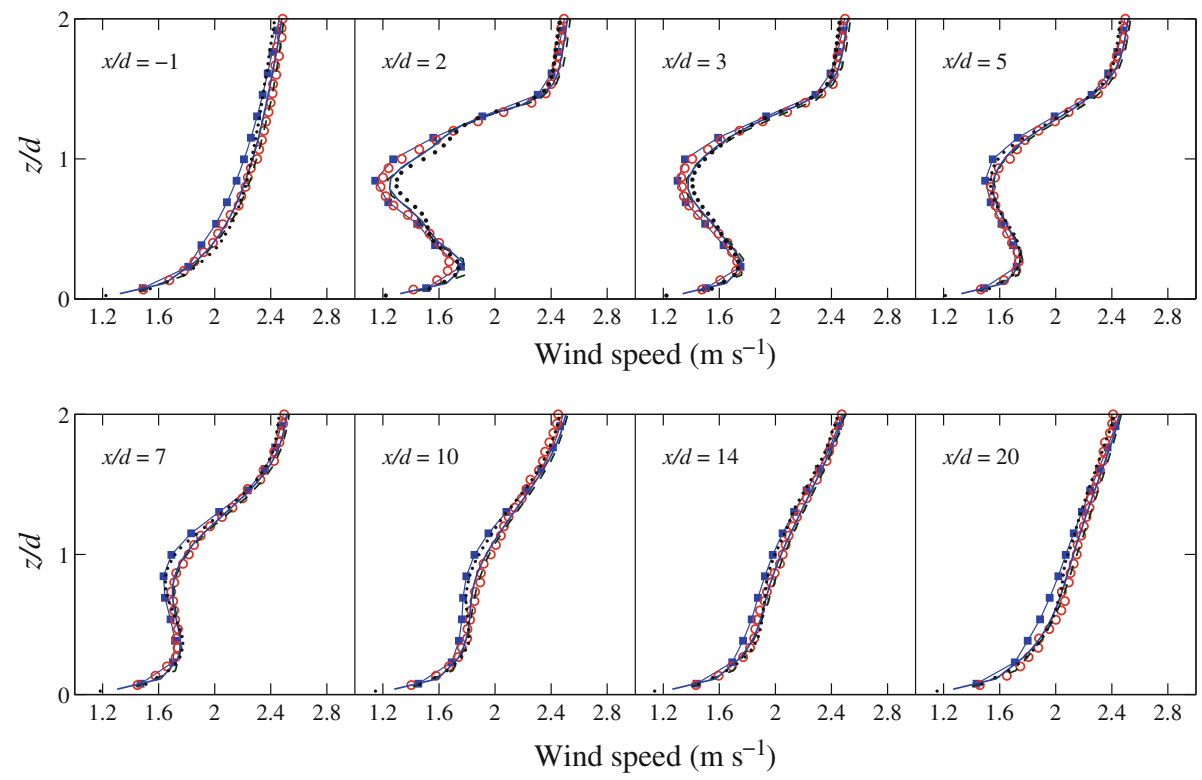

Fig. 12 Comparison of vertical profiles of the time-averaged streamwise velocity $\bar{u}\left(\mathrm{~m} \mathrm{~s}^{-1}\right)$ : wind-tunnel measurements (open circle), ADM-R1 (dotted line), ADM-R2 (solid line), ADM-R3 (dashed line) and ADM-R4 (square, solid line)

In order to illustrate the resolution sensitivity of the computational results, simulations with the ADM-R were performed using four different grid resolutions (see Table 1). Vertical profiles of the streamwise mean velocity and resolved normalised turbulence intensity are presented in Figs. 12 and 13, respectively. The results are compared with the wind-tunnel measurements. From Fig. 12, the incoming velocity profiles $(x / d=-1)$ show very close agreement with the experimental data. In the wake velocity distribution, for the four grid resolutions under consideration, the LES runs with the ADM-R present small sensitivity to grid resolution in the both near-wake and far-wake regions. Simulation results of the turbulence intensity obtained using the four grid resolutions are almost identical in the far-wake region. However, the result obtained from the ADM-R4 case (with 7 by 7 grid points covering the rotor diameter in both the spanwise and vertical directions) starts to show an underes- 

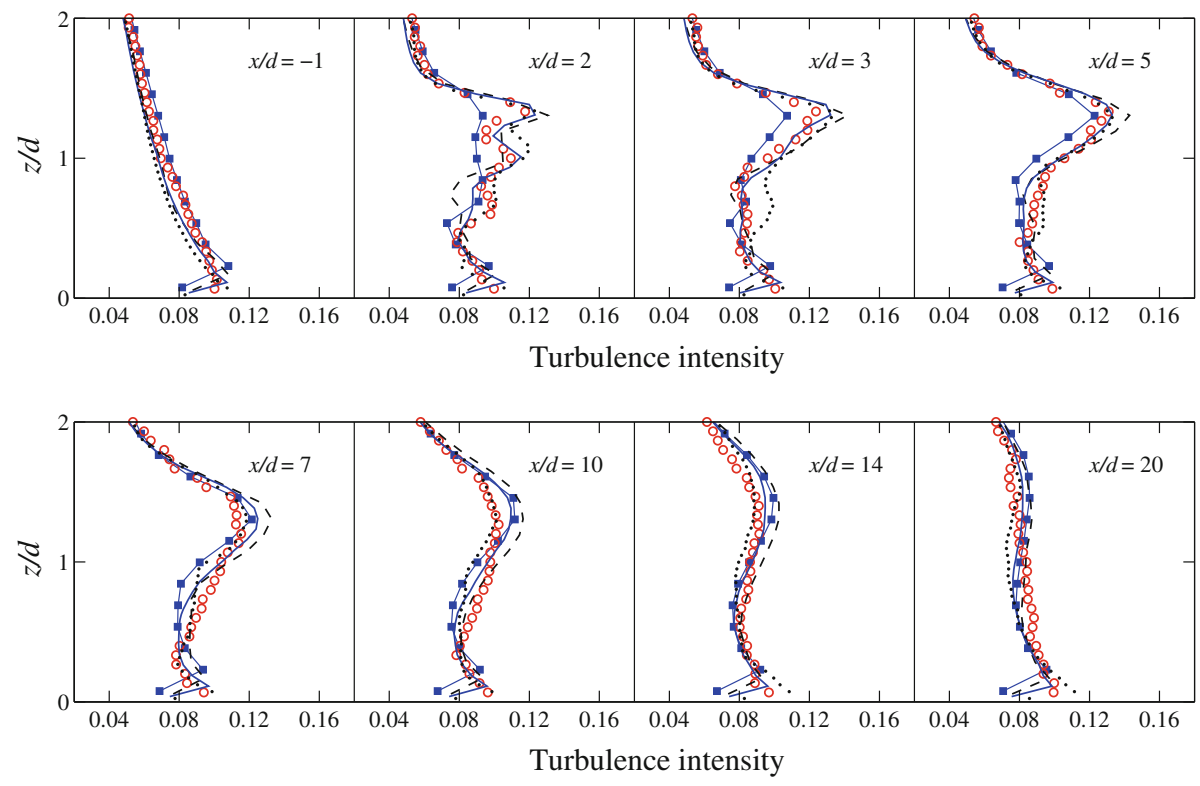

Fig. 13 Comparison of vertical profiles of the streamwise turbulence intensity $\sigma_{u} / \bar{u}_{h u b}$ : wind-tunnel measurements (open circle), ADM-R1 (dotted line), ADM-R2 (solid line), ADM-R3 (dashed line) and ADM-R4 (square, solid line)

timation of the maximum turbulence intensity at the top-tip level in the near-wake region $(x / d=2$ and 3). In summary, our results indicate that the LES framework presented here is capable of accurately reproducing wind-turbine wake characteristics (including mean velocity, turbulence intensity and turbulence fluxes) with very little resolution dependence as long as the at least eight grid points are used to cover the rotor diameter in the simulations.

Figure 14 shows some average trajectories of the simulated flow as it goes through four selected locations in the rotor disk and it moves downwind of the turbine. Also shown in that figure are five cross sections of spanwise velocity vectors. The ADM-R results (Fig. 14b) show evidence of flow rotation in the realistic helicoidal flow trajectories and the spanwise velocity vectors. The intensity of the rotation, induced by the tangential component of the turbine-induced forces, decays with distance downwind from the turbine. The ADM-NR (Fig. 14a), which only computes the thrust force (acting in the x-direction), leads to artificially quasi-straight flow trajectories and a lack of flow rotation.

The dynamically calculated values of the model coefficient $C_{S}$ and scale-dependence parameter $\beta$ obtained using the Lagrangian scale-dependent dynamic model are presented in Fig. 15. As expected, the model coefficient and scale-dependence parameter decrease as the distance to the surface decreases in order to account for the reduction in the characteristic scale of the turbulence near the surface. Moreover, in the near-wake region, an obvious reduction in the model coefficient values distributes from the top tip edge of the turbine to the downstream wake region. This behaviour is consistent with the strong shear and flow anisotropy found at the top tip level in the near-wake region. The Lagrangian scale-dependent dynamic model is able to account for the effects of increased shear and local anisotropy on the reduction of the characteristic scales of the turbulence by dynamically (without any tuning) decreasing the model coefficient. Furthermore, in Fig. 15b, the values of $\beta$ are smaller in the near-wake region and near the surface, where the flow is more anisotropic and the grid size 

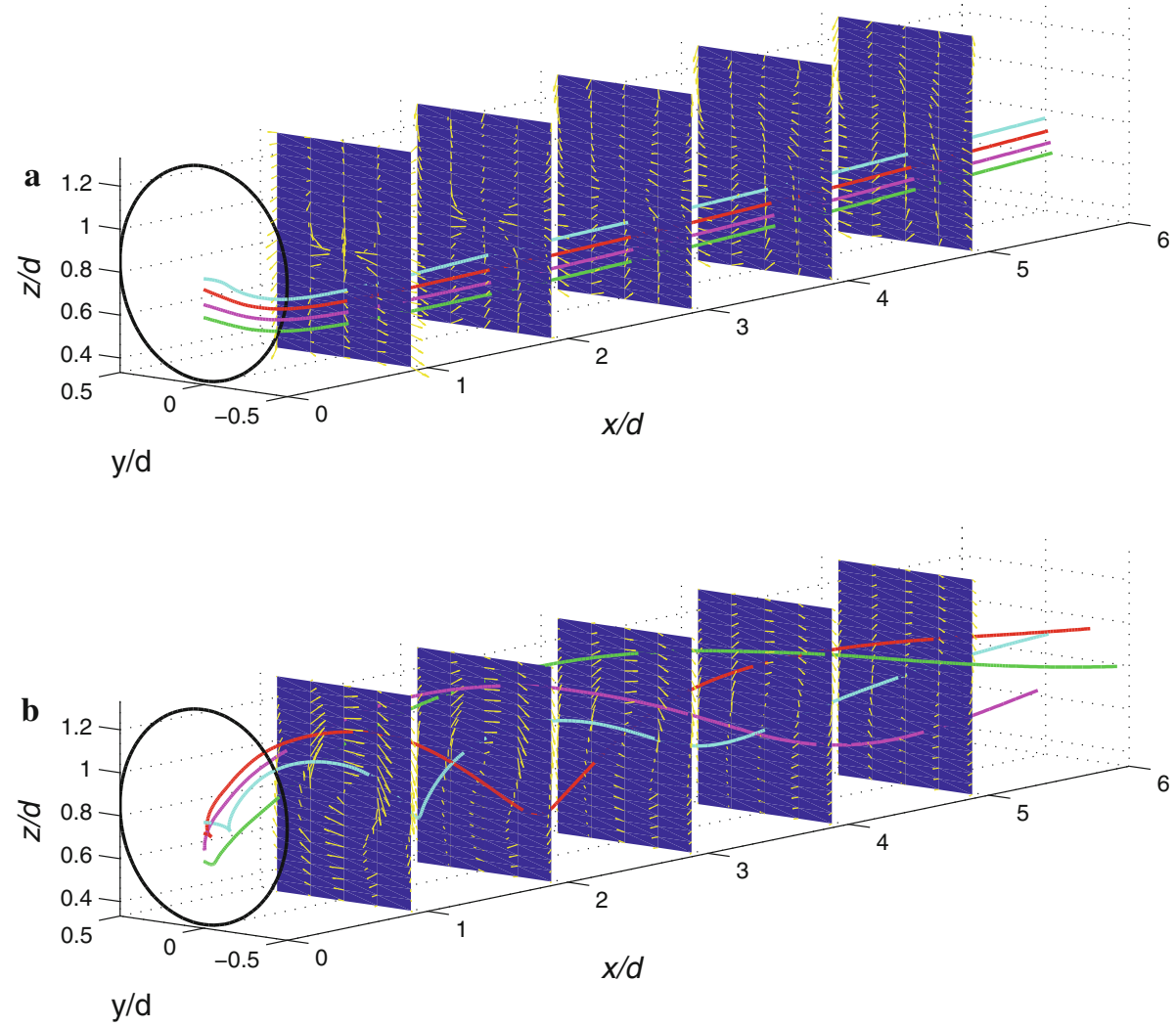

Fig. 14 Vector plots of time-averaged flow velocities $(\bar{v}, \bar{w})$ at different cross sections from ADM-NR (top) and ADM-R (bottom). Colour lines represent streamlines in the near-wake region
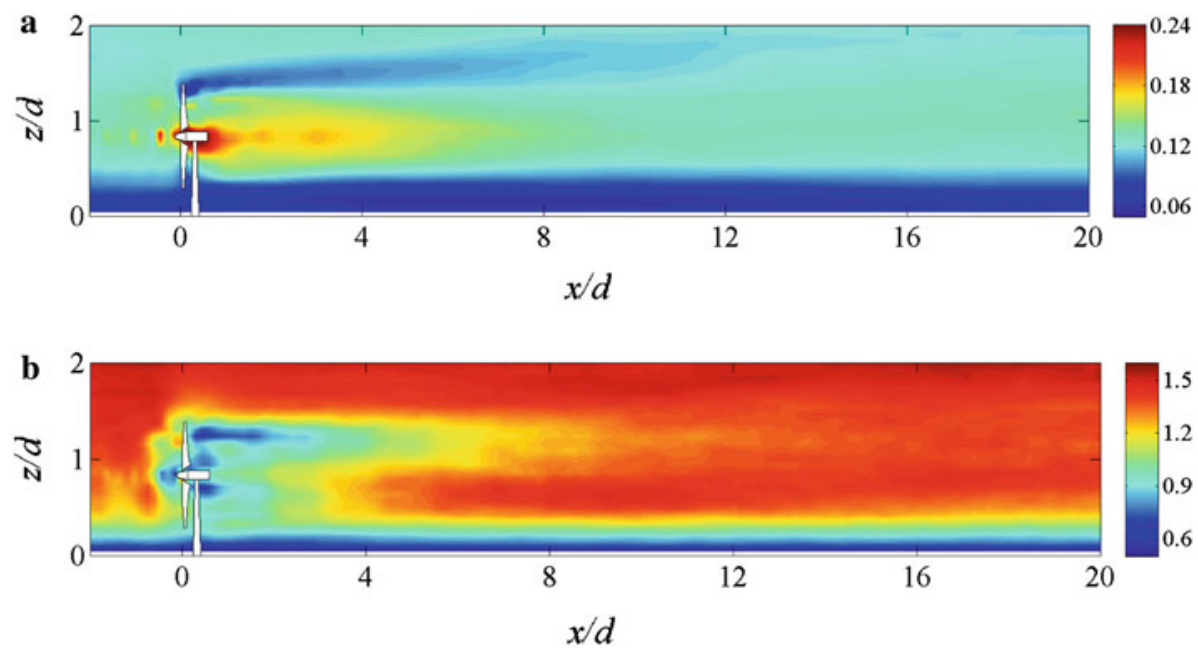

Fig. 15 Contours of a Smagorinsky coefficient $C_{S}$ and $\mathbf{b}$ scale-dependence parameter $\beta$ obtained with the Lagrangian scale-dependent dynamic model 
is likely to fall near the upper edge or even outside of the inertial subrange. In that situation, the Smagorinsky coefficient is expected to depend on resolution and, consequently, $\beta<1$.

\section{Summary}

Large-eddy simulation, coupled with a wind-turbine parametrization, has been used to simulate the wake of a wind turbine developed in a neutrally-stratified turbulent boundary-layer flow. The tuning-free Lagrangian scale-dependent dynamic SGS model (Stoll and Porté-Agel 2006) is used for the parametrization of the SGS stresses. The turbine-induced forces are parametrized using two different actuator-disk models, which distribute the forces over the rotor disk. The ADM-NR calculates the thrust force based on the one-dimensional momentum theory and distributes it uniformly over the disk (Jimenez et al. 2007). The ADM-R uses blade-element theory, together with the local characteristics of the aerofoil (chord length, pitch angle and cross-section geometry), to calculate the lift and drag forces at each location of the rotor. Therefore, unlike the ADM-NR that only computes the thrust force, the ADM-R leads to forces that induce rotation on the flow. Moreover, it yields a non-uniform distribution of the forces. Simulation results obtained with the LES and both actuator-disk models are compared with turbulence statistics obtained from high-resolution velocity measurements collected with hot-wire anemometry in the wake of a miniature wind turbine at the St. Anthony Falls Laboratory atmospheric boundary-layer wind tunnel.

The comparison with the wind-tunnel measurements shows that the turbulence statistics obtained with LES and the ADM-NR have substantial differences with respect to the measurements in the near-wake region. In particular, the model overestimates the average velocity in the centre of the wake, while underestimating the turbulence intensity at the top-tip level, where turbulence levels are highest due to the presence of a strong shear layer. The ADM-R yields more accurate predictions of the different turbulence statistics in the near-wake region. This highlights the importance of the turbine model to induce the rotation of the wake and allow for a non-uniform distribution of the turbine-induced forces. In the far wake, both models produce reasonable results. Besides, our results show that the LES framework presented here is capable of accurately reproducing wind-turbine wake characteristics (including mean velocity, turbulence intensity and turbulence fluxes) with very little resolution dependence as long as the at least eight grid points are used to cover the rotor diameter in the simulations.

The Lagrangian scale-dependent dynamic model (Porté-Agel et al. 2000; Stoll and PortéAgel 2006) is able to dynamically (without any parameter tuning) capture both the spatial variability as well as the scale dependence of the Smagorinsky coefficient using information of the smallest resolved scales. As expected, the value of the model coefficient $C_{S}$ and the scale-dependence parameter $\beta$ decrease near the ground and in the near-wake shear layers in order to account for the reduced characteristic scales of the turbulence and the increased flow anisotropy.

Future research will focus on testing the proposed LES framework under thermallystratified flow conditions, where a dynamic modelling approach can also be used to calculate the SGS heat-flux model coefficient (Porté-Agel 2004; Stoll and Porté-Agel 2006). In addition, we will test the performance of the actuator-line approach, which can be used to distribute the turbine-induced forces along lines that follow the position of the turbine blades. Even though this approach is computationally more demanding (it requires higher spatial and temporal resolutions), it can potentially improve the prediction of the flow dynamics in the near-wake region. Particularly, it would generate helicoidal tip vortices, which cannot be captured using an actuator-disk approach. 
Acknowledgements This research was supported by the Swiss National Science Foundation (grant 200021132122), the National Science Foundation (grant ATM-0854766) and NASA (grant NNG06GE256). Computing resources were provided by the Minnesota Supercomputing Institute.

\section{References}

Albertson JD, Parlange MB (1999) Surfaces length scales and shear stress: implications for land-atmosphere interactions over complex terrain. Water Resour Res 35:2121-2132

Alinot C, Masson C (2002) Aerodynamic simulations of wind turbines operating in atmospheric boundary layer with various thermal stratifications. A collection of the 2002 ASME wind energy symposium technical papers, pp 206-215

Ammara I, Leclerc C, Masson C (2002) A viscous three-dimensional differential/actuator-disk method for the aerodynamic analysis of wind farms. J Sol Energy Eng 124:345-356

Bou-Zeid E, Meneveau C, Parlange M (2005) A scale-dependent lagrangian dynamic model for large eddy simulation of complex turbulent flows. Phys Fluids 17:025105

Calaf M, Meneveau C, Meyers J (2010) Large eddy simulation study of fully developed wind-turbine array boundary layers. Phys Fluids 22:015110

Canuto C, Hussaini MY, Qaurteroni A, Zang TA (1988) Spectral methods in fluid dynamics. Springer, New York, $567 \mathrm{pp}$

Canuto VM, Cheng Y (1997) Determination of the smagorinsky-lilly constant $C_{S}$. Phys Fluids 9:1368-1378

Chamecki M, Meneveau C, Parlange MB (2009) Large eddy simulation of pollen transport in the atmospheric boundary layer. J Aerosol Sci 40:241-255

Chamorro L, Porté-Agel F (2009) A wind-tunnel investigation of wind-turbine wakes: boundary-layer turbulence effects. Boundary-Layer Meteorol 132:129-149

Chamorro L, Porté-Agel F (2010) Effects of thermal stability and incoming boundary-layer flow characteristics on wind-turbine wakes: a wind-tunnel study. Boundary-Layer Meteorol 136:515-533

Deadorff JW (1971) On the magnitude of the subgrid-scale eddy coefficient. J Comput Phys 7:120-133

Deadorff JW (1980) Stratocumulus-capped mixed layers derived from a three-dimensional model. BoundaryLayer Meteorol 18:495-527

Germano M, Piomelli U, Moin PWC (1991) A dynamic subgrid-scale eddy viscosity model. Phys Fluids 7:1760-1765

Ghosal S, Lund TS, Moin P, Akselvoll K (1995) A dynamic localization model for large-eddy simulation of turbulent flows. J Fluid Mech 286:229-255

Glauert H (1935) Aerodynamic theory, vol 4. Springer, Berlin, pp 169-360

Gómez-Elvira R, Crespo A, Migoya E, Manuel F, Hernández J (2005) Anisotropy of turbulence in wind turbine wakes. J Wind Eng Ind Aerodyn 93:797-814

Hau E (2000) Wind turbines: fundamentals, technologies, application, economics. Springer, New York, 783 pp

Horiuti K (1993) A proper velocity scale for modelling subgrid scale eddy viscosities in large-eddy simulation. Phys Fluids 1:146-157

Hunt JCR, Stretch DD, Britter RE (1988) Length scales in stably stratified turbulent flows and their use in turbulence models. In: Puttock JS (ed) Stably stratified flows and gas. Dynamics Clarendon Press, Oxford

Ivanell S, Sørensen JN, Mikkelsen R, Henningson D (2009) Analysis of numerically generated wake structures. Wind Energy 12:63-80

Jimenez A, Crespo A, Migoya E, Garcia J (2007) Advances in large-eddy simulation of a wind turbine wake. J Phys Conf Ser 75:012041

Jimenez A, Crespo A, Migoya E, Garcia J (2008) Large-eddy simulation of spectral coherence in a wind turbine wake. Environ Res Lett 3:015004

Kasmi AE, Masson C (2008) An extended $\kappa-\varepsilon$ model for turbulent flow through horizontal-axis wind turbines. J Wind Eng Ind Aerodyn 96:103-122

Kleissl J, Meneveau C, Parlange MB (2003) On the magnitude and variability of subgrid-scale eddy-diffusion coefficients in the atmospheric surface layer. J Atmos Sci 60:2372-2388

Lilly DK (1992) A proposed modification of the Germano subgrid-scale closure method. Phys Fluids 4(3):633-635

Manwell J, McGowan J, Rogers A (2002) Wind energy explained: theory, design and application. Wiley, New York, $577 \mathrm{pp}$

Masson C, Ammara I, Paraschivoiu I (1997) An aerodynamic method for the analysis of isolated horizontalaxis wind turbines. Int J Rotat Mach 3:21-32 
Medici D, Alfredsson P (2006) Measurement on a wind turbine wake: 3d effects and bluff body vortex shedding. Wind Energy 9:219-236

Meneveau C, Lund T, Cabot W (1996) A lagrangian dynamic subgrid-scale model of turbulence. J Fluid Mech 319:353-385

Mikkelsen R (2003) Actuator disc methods applied to wind turbines. PhD Dissertation,Technical University of Denmark

Moeng C (1984) A large-eddy simulation model for the study of planetary boundary-layer turbulence. J Atmos Sci 46:2311-2330

Phillips AB, Turnock SR, Furlong M (2009) Evaluation of manoeuvring coefficients of a self-propelled ship using a blade element momentum propeller model coupled to a Reynolds averaged Navier Stokes flow solver. Ocean Eng 36:1217-1225

Porté-Agel F (2004) A scale-dependent dynamic model for scalar transport in large-eddy simulations of the atmospheric boundary layer. Boundary-Layer Meteorol 112:81-105

Porté-Agel F, Meneveau C, Parlange MB (2000) A scale dependent dynamic model for large-eddy simulations: application to a neutral atmospheric boundary layer. J Fluid Mech 415:261-284

Porté-Agel F, Meneveau C, Parlange MB, Eichinger WE (2001) A priori field study of the subgrid-scale heat fluxes and dissipation in the atmospheric surface layer. J Atmos Sci 58:2673-2698

Porté-Agel F, Pahlow M, Meneveau C, Parlange MB (2001) Atmospheric stability effect on subgrid-scale physics for large-eddy simulation. Adv Water Resour 24:1085-1102

Schetz JA, Fuhs AE (1996) Handbook of fluid dynamics and fluid machinery. Wiley, New York, 2776pp

Smagorinsky J (1963) General circulation experiments with the primitive equations: I. The basic experiment. Mon Weather Rev 91:99-164

Sørensen JN, Kock CW (1995) A model for unsteady rotor aerodynamics. J Wind Eng Ind Aerodyn 58: 259-275

Sørensen JN, Shen WZ (2002) Numerical modeling of wind turbine wakes. J Fluids Eng 124:393-399

Sørensen JN, Shen WZ, Mundate X (1998) Analysis of wake states by a full-field actuator disc model. Wind Energy 1:73-88

Stoll R, Porté-Agel F (2006) Dynamic subgrid-scale models for momentum and scalar fluxes in large-eddy simulations of neutrally stratified atmospheric boundary layers over heterogeneous terrain. Water Resour Res 42:w01409

Sunada S, Sakaguchi A, Kawachi K (1997) Airfoil section characteristics at a low reynolds number. J Fluids Eng 119:129-135

Troldborg N, Sørensen JN, Mikkelsen R (2007) Actuator line simulation of wake of wind turbine operating in turbulent inflow. J Phys Conf Ser 75:012063

Tseng Y, Meneveau C, Parlange MB (2006) Modeling flow around bluff bodies and predicting urban dispersion using large eddy simulation. Environ Sci Technol 40:2653-2662

Vermeer LJ, Sørensen JN, Crespo A (2003) Wind turbine wake aerodynamics. Prog Aerosp Sci 39:467-510

Wan F, Porté-Agel F (2010) Large-eddy simulation of stably-stratified flow over a steep hill. Boundary-Layer Meteorol. doi:10.1007/s10546-010-9562-4

Wan F, Porté-Agel F, Stoll R (2007) Evaluation of dynamic subgrid-scale models in large-eddy simulations of neutral turbulent flow over a two-dimensional sinusoidal hill. Atmos Environ 41:2719-2728 